\title{
Kullback-Leibler and relative Fisher information as descriptors of locality
}

\author{
H. Levämäki ${ }^{1,2}$, Á. $\mathrm{Nagy}^{3}$, I. Vilja ${ }^{1,4}$, K. Kokko ${ }^{1,2}$ and L. Vitos ${ }^{5,6,7}$ \\ 1 Department of Physics and Astronomy, \\ University of Turku, FI-20014 Turku, Finland \\ 2 Turku University Centre for Materials and Surfaces (MatSurf), Turku, Finland \\ 3 Department of Theoretical Physics, \\ University of Debrecen, H-4002 Debrecen, Hungary \\ 4 Turku Center for Quantum Physics, Turku, Finland \\ 5 Applied Materials Physics, Department of Materials Science and Engineering, \\ Royal Institute of Technology, Stockholm SE-100 44, Sweden \\ ${ }^{6}$ Institute for Solid State Physics and Optics, \\ Wigner Research Centre for Physics, Hungarian Academy of Sciences, \\ P.O. Box 49, H-1525 Budapest, Hungary and \\ ${ }^{7}$ Department of Physics and Astronomy, \\ Division of Materials Theory, Uppsala University, \\ Box 516, SE-75121 Uppsala, Sweden
}

(Dated: October 30, 2017)

\begin{abstract}
Kullback-Leibler and relative Fisher information functionals are applied in studying deviation from local density approximation. The reduced density gradient $s$ and the local kinetic energy parameter $\alpha$ are key ingredients of these new locality descriptors. The relative Kullback-Leibler information density contains extra knowledge as it is negative where the given probability density is smaller than the reference density. The relative Fisher information incorporates the highest order deviations from the uniform electron gas approximation.
\end{abstract}




\section{INTRODUCTION}

Nowadays density functional theory is an essential tool of studying materials. As the exact form of the exchange-correlation functional is not known, constructing high quality approximate functionals is of great importance. Lots of efforts have been paid to develop improved functionals and explore the properties of the exact and approximate functionals. As the local density approximation (LDA) often lacks the desired accuracy for numerical calculations, nonlocal functionals have been designed. There is(are) other ingredient(s) in these functionals besides the electron density. In the generalized gradient approximations (GGA) the dimensionless reduced gradient density $s$ has a decisive role in addition to the density. Going beyond the GGAs another crucial dimensionless quantity $\alpha$ is defined from kinetic energy densites. These carefully constructed dimensionless quantities have received much attention. However, according to our knowledge, no one observed that these locality descriptors are also key ingredients of relative information measures. The present analysis intends to shed light on these quantities utilized in approximate exchange-correlation functionals using Kullback-Leibler and relative Fisher information functionals.

Information theoretical concepts have proved to be beneficial in analyzing physical systems. They have been applied, among others, in density functional theory (see e. g. [1-3]). These ideas have found applications for example in studying atoms and molecules [2-19]. Our aim is to give a new insight in analysing density functionals via relative information. The advance of the present work over the current state of knowledge in density functionals is the inclusion of relative information concepts. It might have an impact on new functional constructions as these information functionals provide very sensitive measures of the deviations from the local density approximation. It has now turned out that the relative Kullback-Leibler information density contains extra knowledge as it is negative where the given probability density is smaller than the reference probability density. On the other hand, since the relative Fisher information contains derivatives, it detects even higher order deviations from the uniform electron gas approximation. Both local and global descriptors are presented.

In the following section the definitions of the Kullback-Leibler and relative Fisher information are summarized. Locality descriptors of functionals are reviewed in the subsequent section. Then it is shown that these locality descriptors are key ingredients of relative 
information. The final section is devoted to a comparison of descriptors and discussion.

\section{KULLBACK-LEIBLER AND RELATIVE FISHER INFORMATION}

Consider a probability density function $f(\mathbf{r})$. Shannon information is defined as [20]

$$
S_{f}=-\int f(\mathbf{r}) \ln f(\mathbf{r}) d \mathbf{r} .
$$

Having two probability density functions the information gain we can obtain using $f(\mathbf{r})$, instead of the other (reference) probability density function $f^{r e f}(\mathbf{r})$, is given by the relative or Kullback-Leibler information (also called cross-entropy) [21]

$$
G=\int f(\mathbf{r}) \ln \frac{f(\mathbf{r})}{f^{r e f}(\mathbf{r})} d \mathbf{r} .
$$

The relative information is a measure of the deviation of $f(\mathbf{r})$ from the reference density $f^{r e f}(\mathbf{r}) . G$ is never negative [21]:

$$
G \geq 0
$$

We have $G=0$ if and only if the probability density function $f$ equals the reference function $f^{r e f}$ almost everywhere.

The Fisher information [22] measures the information that we can obtain for the parameter $\theta$ of the distribution function $f(x \mid \theta)$. It is defined as

$$
I_{f}(\theta)=\int f(x \mid \theta)\left[\frac{\partial \ln f(x \mid \theta)}{\partial \theta}\right]^{2} d x=\int \frac{\left[f^{\prime}(x \mid \theta)\right]^{2}}{f(x \mid \theta)} d x .
$$

For example, we want to measure the position of a particle. Let $\theta$ be this position and we try to estimate this quantity. Suppose that we observe the particle at the position $x$. The fluctuation of the position observed should be translation invariant:

$$
f(x \mid \theta)=f(x-\theta)=f(\gamma)
$$

Generally, $\theta$ stands for any physical quantity that we intend to measure. The fluctuation of the observed and the true values of the physical quantity is always supposed to be indepen-

dent of the value of $\theta$. If relation (5) holds, $\theta$ is a parameter of locality [23, 24]. Then we obtain that

$$
\frac{\partial f(x \mid \theta)}{\partial \theta}=\frac{\partial f(x-\theta)}{\partial(x-\theta)}=-\frac{\partial f(\gamma)}{\partial \gamma}
$$


That is, the Fisher information (4)

$$
I_{f}(\theta)=\int\left[\frac{\partial f(x-\theta)}{\partial(x-\theta)}\right]^{2} / f(x-\theta) d x .
$$

does not depend on $\theta$. Therefore, the parameter $\theta$ is taken to be zero:

$$
I_{f}=\int \frac{\left[f^{\prime}(x)\right]^{2}}{f(x)} d x .
$$

For a normal distribution

$$
f(x)=\frac{1}{\sqrt{2 \pi \sigma^{2}}} e^{-\frac{(x-\mu)^{2}}{2 \sigma^{2}}}
$$

with mean $\mu$ and variance $\sigma^{2}$, the Fisher information is

$$
I_{f}=\frac{1}{\sigma^{2}}
$$

Generally, the Cramer-Rao inequality holds, that is, the variance is bounded from below by the reciprocal of the Fisher information [23]

$$
\operatorname{Var}(x) \geq \frac{1}{I_{f}} .
$$

$I_{f}$ estimates intrinsic accuracy, it shows the 'narrowness' of the distribution function $f$. For a three variable function the analogue of Eq. (8) can be written as

$$
I=\int \frac{|\nabla f(\mathbf{r})|^{2}}{f(\mathbf{r})} d \mathbf{r}
$$

which is the trace of the Fisher information matrix. The relative Fisher information corresponding Eq. (12) is defined as [25]

$$
J=\int f(\mathbf{r})\left|\nabla\left(\ln \frac{f(\mathbf{r})}{f^{r e f}(\mathbf{r})}\right)\right|^{2} d \mathbf{r} .
$$

It can be rewritten as

$$
J=\int f(\mathbf{r})\left|\frac{\nabla f(\mathbf{r})}{f(\mathbf{r})}-\frac{\nabla f_{r e f}(\mathbf{r})}{f^{r e f}(\mathbf{r})}\right|^{2} d \mathbf{r} .
$$

There is a simple relationship between the Shannon and Fisher information [17]. Nagy and March [26] introduced the ratio of the density gradient to the electron density $n$ as a local wave-number to characterize the ground state of atoms and molecules. Independently, 
Kohout, Savin and Preuss [27] also investigated the role of the quantity $|\nabla n / n|$ in the shell structure of atoms. With the present notation the local wave-vector is

$$
\mathbf{q}(\mathbf{r})=-\frac{\nabla f(\mathbf{r})}{f(\mathbf{r})}
$$

In estimation theory $-\mathbf{q}(\mathbf{r})$ is called the linear score function [28]. With the concepts of the local Shannon information $s(\mathbf{r})=-f(\mathbf{r}) \ln f(\mathbf{r})$ and the local Fisher information $i(\mathbf{r})=|\nabla f(\mathbf{r})|^{2} / f(\mathbf{r})$ introduced earlier [17]

$$
\mathbf{q}(\mathbf{r})=\nabla\left(\frac{s(\mathbf{r})}{f(\mathbf{r})}\right)
$$

and

$$
(q(\mathbf{r}))^{2}=\frac{i(\mathbf{r})}{f(\mathbf{r})}
$$

That is, the local wave-vector $\mathbf{q}(\mathbf{r})$ is the gradient of the function $s(\mathbf{r}) / f(\mathbf{r})$, - the expectation value of which is the Shannon information - and the square of the local wave-vector is $i(\mathbf{r}) / f(\mathbf{r})$ - the expectation value of which is the Fisher information. The latter can be also expressed as that the variance of the linear score function is the Fisher information.

Now we introduce the relative local wave-vector as

$$
\tilde{\mathbf{q}}(\mathbf{r})=\mathbf{q}(\mathbf{r})-\mathbf{q}^{r e f}(\mathbf{r})
$$

with

$$
\mathbf{q}^{r e f}(\mathbf{r})=-\frac{\nabla f^{r e f}(\mathbf{r})}{f^{r e f}(\mathbf{r})}
$$

$\tilde{\mathbf{q}}(\mathbf{r})$ corresponds to the linear score function of the pair relative to $f^{r e f}(\mathbf{r})$. Then the relation between the Kullback-Leibler and the relative Fisher information has the form

$$
\tilde{\mathbf{q}}(\mathbf{r})=\nabla\left(\frac{g(\mathbf{r})}{f(\mathbf{r})}\right)
$$

and

$$
(\tilde{q}(\mathbf{r}))^{2}=\frac{j(\mathbf{r})}{f(\mathbf{r})}
$$

The Kullback-Leibler and the relative Fisher information densities are defined as

$$
g(\mathbf{r})=f(\mathbf{r}) \ln \frac{f(\mathbf{r})}{f^{r e f}(\mathbf{r})}
$$


and

$$
j(\mathbf{r})=f(\mathbf{r})\left|\nabla\left(\ln \frac{f(\mathbf{r})}{f^{r e f}(\mathbf{r})}\right)\right|^{2},
$$

respectively. We can also see that relative Fisher information is the variance of the linear score function of the pair relative to $f^{r e f}(\mathbf{r})$.

\section{LOCALITY DESCRIPTORS OF FUNCTIONALS}

The exact density functional is unknown, the exchange-correlation energy needs to be approximated. The simplest approximation is the local density approximation (LDA)

$$
E_{x c}^{L D A}=\int d \mathbf{r} n(\mathbf{r}) \epsilon_{x c}^{u n i f}(n(\mathbf{r})),
$$

where $\epsilon_{x c}^{u n i f}(\mathbf{r})=\epsilon_{x}^{u n i f(\mathbf{r})}+\epsilon_{c}^{u n i f}(\mathbf{r})$ is the exchange-correlation energy per electron of the electron gas with uniform density $n$.

The simplest semilocal approximation is the generalized gradient approximation (GGA):

$$
E_{x c}^{G G A}[n]=\int d \mathbf{r} n \epsilon_{x}^{u n i f}(n) F_{x c}^{G G A}(n, s),
$$

where $\epsilon_{x}^{\text {unif }}(\mathbf{r})=-(3 / 4)(3 n(\mathbf{r}) / \pi)^{1 / 3}$ is the exchange energy per electron of the jellium and

$$
s(\mathbf{r})=\frac{|\nabla n(\mathbf{r})|}{2\left(3 \pi^{2}\right)^{1 / 3} n(\mathbf{r})^{4 / 3}} .
$$

$s(\mathbf{r})$ is the reduced density gradient, an important dimensionless parameter measuring the inhomogenity of the density. Atomic units are used in the paper. The enhancement factor can be partitioned as

$$
F_{x c}^{G G A}(n, s)=F_{x}^{G G A}(s)+F_{c}^{G G A}(n, s)
$$

The high-density limit of $F_{x c}^{G G A}$ is the exchange enhancement factor $F_{x}^{G G A}$. In case of uniform density $\left(F_{x}^{G G A}(s=0)=F_{x}^{L D A}=1\right)$ the LDA is recovered.

In the semilocal meta-GGA another ingredient, the kinetic energy density

$$
\tau(\mathbf{r})=2 \sum_{i}^{\text {occup }}\left|\nabla \psi_{i}(\mathbf{r})\right|^{2} / 2
$$

is involved. $\psi_{i}(\mathbf{r})$ is a Kohn-Sham orbital. The exchange-correlation energy can be written as

$$
E_{x c}^{M G G A}[n]=\int d \mathbf{r} n \epsilon_{x}^{u n i f}(n) F_{x c}^{M G G A}(n, s, \alpha)
$$


where

$$
\alpha=\frac{\tau-\tau^{W}}{\tau^{u n i f}}
$$

$\tau^{W}=|\nabla n(\mathbf{r})|^{2} /(8 n(\mathbf{r}))$ and $\tau^{u n i f}=(3 / 10)\left(3 \pi^{2}\right)^{2 / 3} n^{5 / 3}(\mathbf{r})$ are the Weizsäcker and the uniform kinetic energy densities, respectively. As the kinetic energy density can be partitioned as the sum of the Weizsäcker and the Pauli terms

$$
\tau=\tau^{W}+\tau^{\text {Pauli }}
$$

where $\tau^{\text {Pauli }}$ is defined by this equation. The parameter $\alpha$ can be rewritten as

$$
\alpha=\frac{\tau^{\text {Pauli }}}{\tau^{\text {unif }}}
$$

$\alpha$ is a useful quantity as it can distinguish between covalent single $(\alpha \approx 0)$, metallic $(\alpha \approx 1)$ and weak $(\alpha>>1)$ bonds [29-33].

\section{DESCRIPTORS $\alpha$ AND $s$ AS KEY INGREDIENTS OF RELATIVE INFOR- MATION}

The parameter $\alpha$ is defined as the ratio of the Pauli and the uniform kinetic energy densities (Eq. (32)). To use $\alpha$ in the Kullback-Leibler information we have to express it with probability density-like function. Therefore we introduce two distribution descriptors

$$
p^{u n i f}=\frac{\tau^{u n i f}}{T^{u n i f}}
$$

and

$$
p^{\text {Pauli }}=\frac{\tau^{\text {Pauli }}}{T^{\text {Pauli }}}
$$

where $T^{\text {unif }}$ and $T^{\text {Pauli }}$ are the uniform and Pauli kinetic energies:

$$
T^{u n i f}=\int \tau^{u n i f} d \mathbf{r}
$$

and

$$
T^{\text {Pauli }}=\int \tau^{\text {Pauli }} d \mathbf{r}
$$


which we refer as the uniform and Pauli probabilities. Here we utilize the fact that the Pauli and the uniform kinetic energy densities are non-negative. The uniform probability $p^{\text {unif }}$ is an escort distribution ( see e.g.[34])

$$
p^{u n i f}(\mathbf{r})=\frac{n^{5 / 3}(\mathbf{r})}{\int n^{5 / 3}\left(\mathbf{r}^{\prime}\right) d \mathbf{r}^{\prime}}
$$

Escort distributions are often applied among others in the theory of fractals. They are closely related to Rényi information. We mention in passing that the kinetic energy functional of the uniform electron system can be easily expressed with a Rényi information [35].

The concept of escort distribution can be further generalized [34]:

$$
P(\mathbf{r})=\frac{\phi(\mathbf{r})}{\int \phi\left(\mathbf{r}^{\prime}\right) d \mathbf{r}^{\prime}}
$$

Any properly selected positive test function $\phi[36,37]$ can yield a distribution function. Clearly, the special choice of $\phi=n^{5 / 3}$ leads to the uniform probability $p^{\text {unif }}$. The Pauli probability (Eq. (34)) and the Weizsäcker probability (Eq. (45)) are defined in this general sence.

Define now the Pauli relative information with the uniform kinetic energy density as reference distribution

$$
G^{\text {Pauli }}=\int g^{\text {Pauli }} d \mathbf{r}
$$

where the Pauli relative information density $g^{\text {Pauli }}$ is

$$
g^{\text {Pauli }}=p^{\text {Pauli }} \ln \frac{p^{\text {Pauli }}}{p^{\text {unif }}} .
$$

Substituting Eqs. (33) - (36) into Eq. (40) we are led to

$$
g^{\text {Pauli }}=\frac{\tau^{\text {Pauli }}}{T^{\text {Pauli }}}\left(\ln \alpha+\ln \frac{T^{\text {unif }}}{T^{\text {Pauli }}}\right) .
$$

Then, the Pauli relative information takes the form

$$
G^{\text {Pauli }}=\frac{1}{T^{\text {Pauli }}} \int \tau^{\text {Pauli }} \ln \alpha d \mathbf{r}+\ln \frac{T^{\text {unif }}}{T^{\text {Pauli }}} .
$$

If we consider slowly varying densities, then $\alpha \approx 1, \tau^{\text {Pauli }} \approx \tau^{\text {unif }}$ and $G^{\text {Pauli }} \approx 0$, that is, the local density functional is an appropriate approximation. $g^{\text {Pauli }}$ and $G^{\text {Pauli }}$ can be considered local and global descriptors. These quantities indicate how well the LDA approximation can be applied for the given system. 
We can also define the Weizsäcker relative information with the uniform kinetic energy density as reference distribution

$$
G^{W}=\int g^{W} d \mathbf{r}
$$

where

$$
g^{W}=p^{W} \ln \frac{p^{W}}{p^{u n i f}}
$$

and

$$
p^{W}=\frac{\tau^{W}}{T^{W}}
$$

are the Weizsäcker relative information density and probability, respectively. Substituting Eqs. (33), (44) and (45) into Eq. (43) and taking into account that

$$
\frac{\tau^{W}}{\tau^{\text {unif }}}=\frac{5}{3} s^{2}
$$

we are led to

$$
g^{W}=\frac{\tau^{W}}{T^{W}}\left(\ln s^{2}+\ln \frac{5 T^{u n i f}}{3 T^{W}}\right) .
$$

Then, the Weizsäcker relative information takes the form

$$
G^{W}=\frac{1}{T^{W}} \int \tau^{W} \ln s^{2} d \mathbf{r}+\ln \frac{5 T^{\text {unif }}}{3 T^{W}} .
$$

We arrived at the result that the Pauli and the Weizsäcker relative information densities can be expressed in terms of the logarithm of $\alpha$ and $s$, respectively. Note that $g^{\text {Pauli }}$ and $g^{W}$ can also be negative at some values of $\mathbf{r}$. It can be seen from the definition equations (40) and (44) that negative values appear at the points where the given probability density is smaller than the reference density. Turning to the relative Fisher information we can define the Pauli relative Fisher information and information density as

$$
J^{\text {Pauli }}=\int j^{\text {Pauli }}(\mathbf{r}) d \mathbf{r}
$$

and

$$
j^{\text {Pauli }}(\mathbf{r})=p^{\text {Pauli }}(\mathbf{r})\left|\nabla\left(\ln \frac{p^{\text {Pauli }}(\mathbf{r})}{p^{\text {unif }}(\mathbf{r})}\right)\right|^{2} .
$$


Making use of Eqs. (34) - (36) the relative Fisher information density can be given by

$$
j^{\text {Pauli }}=\frac{\tau^{\text {Pauli }}}{T^{\text {Pauli }}}|\nabla(\ln \alpha)|^{2} .
$$

Define now the Weizsäcker relative Fisher information and the corresponding information density as

$$
J^{W}=\int j^{W}(\mathbf{r}) d \mathbf{r}
$$

and

$$
j^{W}(\mathbf{r})=p^{W}(\mathbf{r})\left|\nabla\left(\ln \frac{p^{W}(\mathbf{r})}{p^{\text {unif }}(\mathbf{r})}\right)\right|^{2} .
$$

Taking into account Eq. (46) we arrive at

$$
j^{W}=\frac{\tau^{W}}{T^{W}}\left|\nabla\left(\ln \left(s^{2}\right)\right)\right|^{2} .
$$

Our final expressions show that the Pauli and the Weizsäcker relative Fisher information densities can be expressed in terms of the gradient of the logarithm of $\alpha$ and $s$, respectively. We emphasize that the Pauli and the Weizsäcker relative information densities are defined with kinetic energy densities. Therefore, the deviation from the local density approximation means deviation of the given kinetic energy density from the LDA kinetic energy density.

It has been shown in [29] that $\alpha$ can recognize all types of typical regions; regions of one and two-electron densities characterizing single bonds: $\alpha \approx 0$; regions having slowly varying densities that are characteristic to metallic bonds: $\alpha \approx 1$; and regions with density overlap between closed shells that is specific in noncovalent bonds: $\alpha>>1$. Both $g^{\text {Pauli }}$ and $j^{\text {Pauli }}$ are expressed with $\alpha$, therefore we anticipate that $g^{\text {Pauli }}$ and $j^{\text {Pauli }}$ will also distinguish between covalent single $(\alpha \approx 0)$, metallic $(\alpha \approx 1)$ and weak $(\alpha>>1)$ bonds. It can be the subject of further research.

\section{COMPARISON OF DESCRIPTORS AND DISCUSSION}

Now, we analyze locality descriptors for some systems. For comparison, we also present results for the electron localization function (ELF) defined as [38]

$$
E L F=\frac{1}{1+\left(\tau^{\text {Pauli }} / \tau^{\text {unif }}\right)^{2}} .
$$



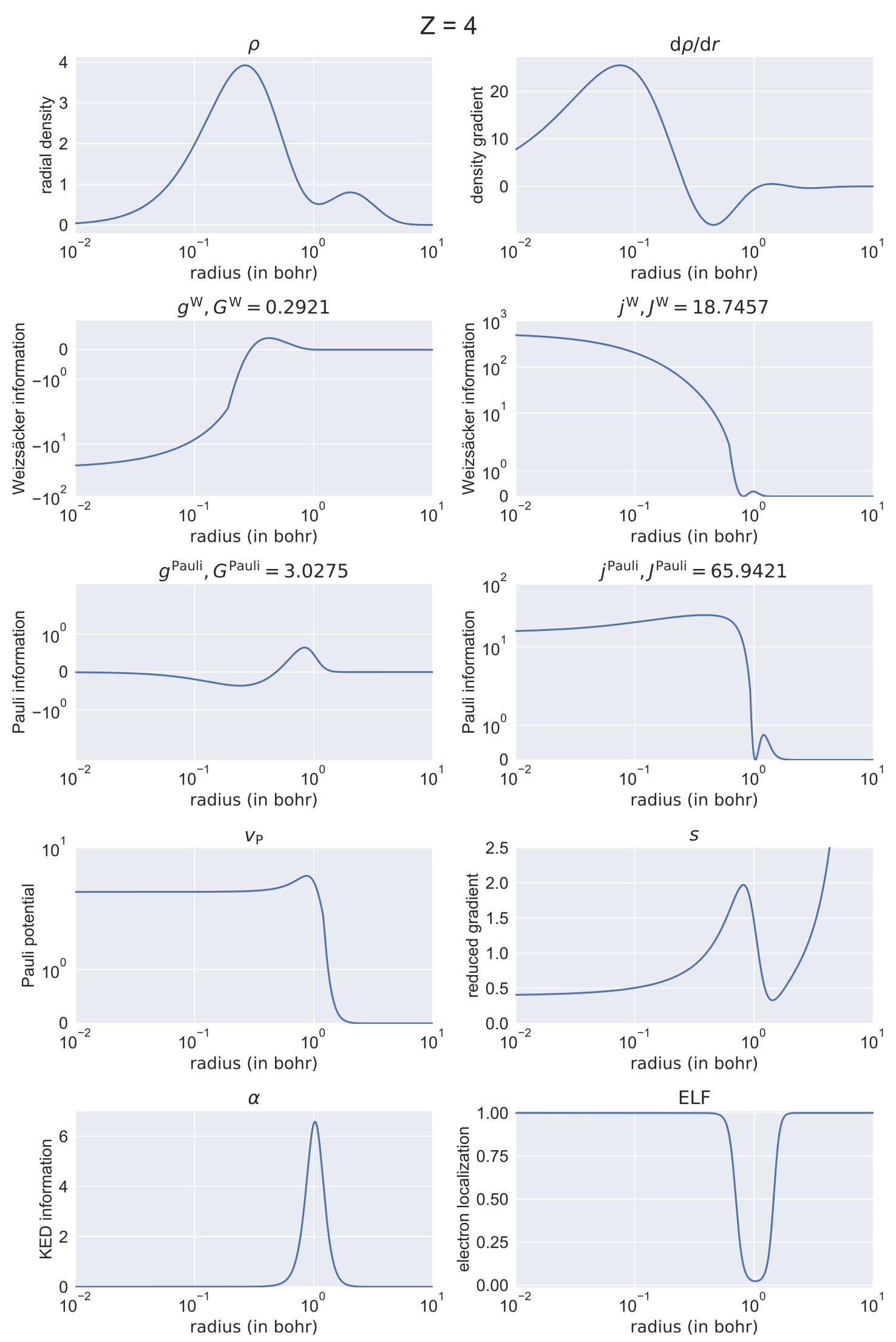

FIG. 1: (Color online) $\varrho, d \varrho / d r, g^{W}, j^{W}, g^{\text {Pauli }}, j^{\text {Pauli }}, v_{P}, s, \alpha$ and $E L F$ as functions of radial distance for the Be atom in atomic units. 
Utilising Eq. (32), ELF can be expressed with $\alpha$ as

$$
E L F=\frac{1}{1+\alpha^{2}} .
$$

$E L F$ has proved to be a very useful quantity for several applications [38-41]. The uniform electron gas is the reference system here. For this model $s=0, \alpha=1, g^{\text {Pauli }}=0, g^{W}=0$, $j^{\text {Pauli }}=0, j^{W}=0$ and $E L F=1 / 2$, where Eqs. (26), (32), (41), (47), (51), and (54) and (56) were used. That is, the relative information densities $g^{\text {Pauli }}, g^{W}, j^{\text {Pauli }}$ and $j^{W}$ measure the deviation from the uniform electron gas approximation.

Consider now atoms and calculate all these descriptors for them. We deliberately chose atomic systems because they are supposed to be far from the homogeneous electron gas and the deviation from LDA can be clearly seen. Further, the shell structure can be nicely detected in atoms. Another quantity, the Pauli potential $v_{P}$ was also determined. The Pauli potential, defined as a functional derivative of the Pauli energy with respect to the density,

$$
v_{P}=\frac{\delta T^{\text {Pauli }}}{\delta n},
$$

is a key quantity. Its appearence is the consequence of the Pauli principle. The Pauli potential clearly shows the shell structure of atoms.

We have implemented all of the descriptors and the Pauli potential in dftatom [42] atomic solver, which we have used in our calculations. The radial Schrödinger equations are integrated numerically without the use of basis sets. To describe exchange and correlation effects we use the Vosko-Wilk-Nusair version of LDA [43]. The gradation and the number of radial mesh points is chosen in such a way that the calculated Kohn-Sham orbital eigenvalues agree to six decimal places with literature [42].

Figs. 1.- 5. present locality descriptors for atoms $\mathrm{Be}, \mathrm{Ne}, \mathrm{Ar}, \mathrm{Kr}$ and Xe. It is also instructive to plot the radial density $\left(\varrho=4 r^{2} \pi n\right)$ and its derivative. The upper row in the figures present the radial density $\varrho$, the relative information densities $g^{\text {Pauli }}, g^{W}, j^{\text {Pauli }}$ and $j^{W}$, while the lower row shows the derivative of the radial density $d \varrho / d r, v_{P}, s, \alpha$ and $E L F$ as a function of the radial distance. All these descriptors manifest the shell structure. Most of the information quantities are positive, but $g^{P a u l i}$ and $g^{W}$ can also be negative. Negative values appear where the given probability density is smaller than the reference density. Therefore $g^{P a u l i}$ and $g^{W}$ incorporate extra information. If we integrate the relative information densities, we obtain global descriptors $G^{\text {Pauli }}$ and $G^{W}$ to characterize 
the deviations from LDA. These global values are reported for each atom in the headers of the figures.

While $s$ involves only the density and the gradient of the density, the other descriptors include the kinetic energy densities, that is, more direct knowledge of the orbital behaviour.

Only $j^{\text {Pauli }}$ and $j^{W}$ involve the gradient of $s$ and $\alpha$, that is, the relative Fisher information measures not the deviation of the kinetic energy density from the reference one, but the deviation of the logarithmic derivative of the kinetic energy density from the reference one. No wonder that one can observe the highest values in the figures of $j^{\text {Pauli }}$ and $j^{W}$. It means that the relative Fisher information incorporates the highest order deviations from LDA. The integrals of $j^{\text {Pauli }}$ and $j^{W}$ give the global quantities $J^{\text {Pauli }}$ and $J^{W}$ specifying the difference from LDA. The high values of $J^{\text {Pauli }}$ and $J^{W}$ clearly reflect that the relative Fisher information is the most sensitive measure of deviations from LDA. All plotted quantities show the shell structure. However, the numbers and the positions of the maxima and the minima of these quantities are different from those of the radial densities. It is the consequence of the definitions of these quantities. Moreover, the local information quantities are related to the kinetic energy densities, so we cannot expect the same behaviour that the radial density exhibits. Not all the shells of atoms that appear in the radial density, are recognizable in all plots. In certain cases the maximum or minimum is too shallow to be noticed in the figures (e.g. in the plots of $g^{w}$.) The relative information densities highlight different parts of the atom than the radial density. Those parts where the Pauli or Weizsäcker kinetic energy densities differ from the uniform kinetic energy density. We have studied the maxima and the minima of the quantities presented. It follows from the definition (55) of ELF that ELF has a maximum (minimum) when $\alpha$ displays a minimum (maximum). The relationships between the extrema of other quantities are more complicated. We found that the radial distance where the radial density has a minimum, is almost the same radial distance where $E L F$ and $j^{\text {Pauli }}$ show a minimum (and $\alpha$ has maximum) in all atoms studied.

The Kullback-Leibler and the relative Fisher information densities are defined in terms of the Pauli, the Weizsäcker and the uniform kinetic energy densities. Therefore, the assertion that these quantities measure the deviations from LDA, means that these concepts show how much the Pauli or the Weizsäcker kinetic energy densities differ from the uniform (Thomas-Fermi) kinetic energy. The parameter $\alpha$ also contains knowledge on the kinetic energy density (versus to the uniform kinetic energy density). Meta-GGA approximations 

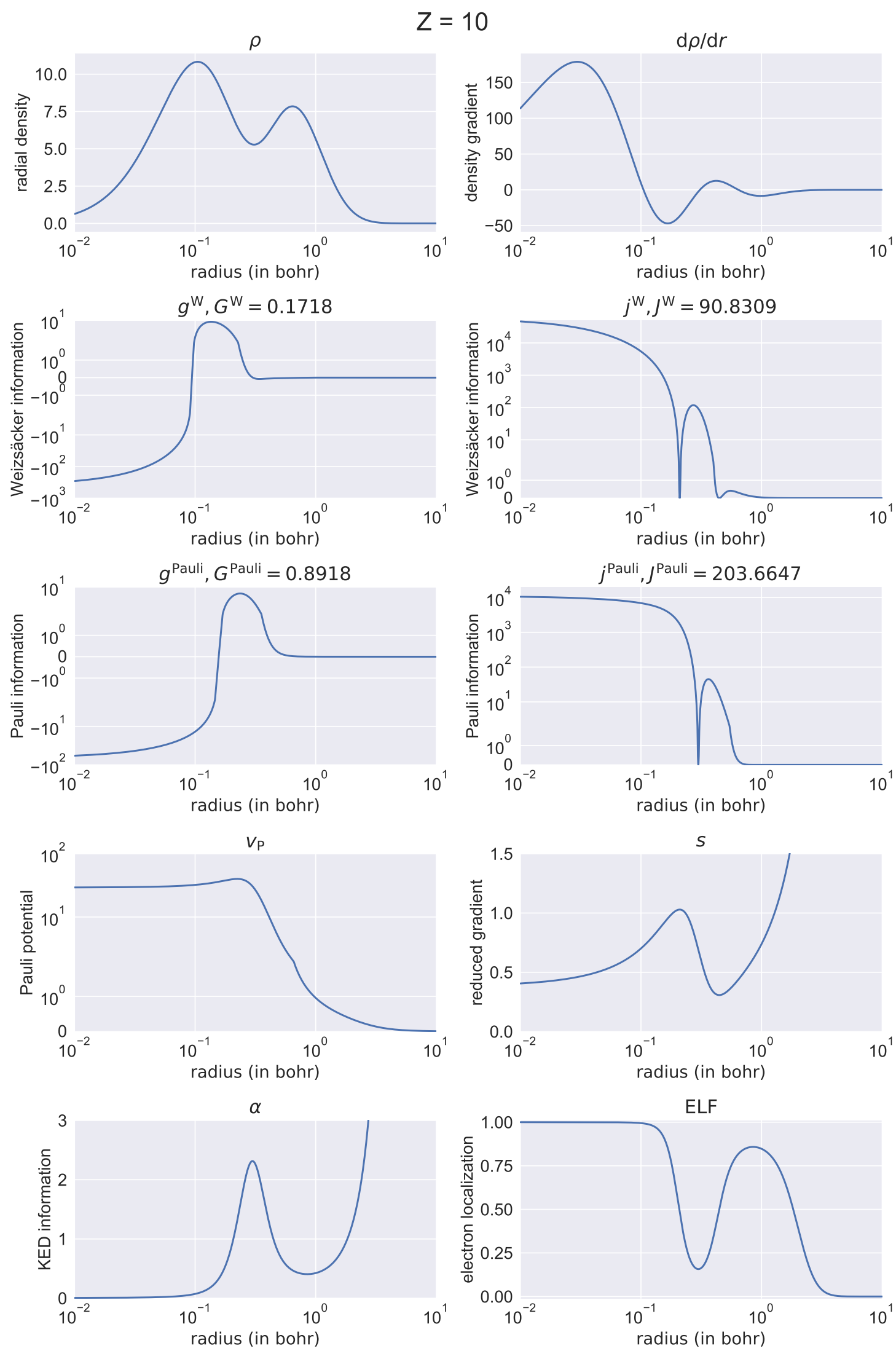

FIG. 2: (Color online) $\varrho, d \varrho / d r, g^{W}, j^{W}, g^{\text {Pauli }}, j^{\text {Pauli }}, g^{\text {Pauli }}, v_{P}, s, \alpha$ and $E L F$ as functions of radial distance for the Ne atom in atomic units. 
for the exchange and correlation use this quantity $\alpha$ incorporating kinetic energy density into the exchange-correlation functional. In this paper we showed how the knowledge on kinetic energy densities can be grasped with information theoretical concepts.

In summary, we have shown that the relative information functionals provide alternative measures of the deviation from local density approximation. The relative Kullback-Leibler information density includes extra knowledge compared to the frequently used local descriptors. The relative Fisher information incorporates the deviation of the logarithmic derivative of the kinetic energy density from the reference one, and therefore it embodies the highest order deviations from LDA.

\section{Acknowledgments}

L.V. acknowledges financial support by the Swedish Research Council, the Swedish Foundation for Strategic Research, the Swedish Foundation for International Cooperation in Research and Higher Education, the Carl Tryggers Foundation, and the Hungarian Scientific Research Fund (OTKA 109570). Á. N. acknowledges the National Research, Development and Innovation Fund of Hungary, financed under 123988 funding scheme. This research was supported by the EU-funded Hungarian grant EFOP-3.6.2-16-2017-00005.

[1] S. B. Sears, R. G. Parr, and U. Dinur, Isr. J. Chem. 1980, 19165.

[2] R. Nalewajski, Chem. Phys. Lett. 2003, 372, 28.

[3] Á. Nagy, J. Chem. Phys. 2003, 119, 9401.

[4] S. R. Gadre, Phys. Rev. A 1984, 30, 620; S. R. Gadre, S. B. Sears,S. J. Chakravorty and R. D. Bendale, Phys. Rev. A 1985, 32, 2602.

[5] M. Hó, R. P. Sagar, V. H. Smith Jr. and R. O. Esquivel, J. Phys. B. 1994, 27, 5149; M. Hó, R. P. Sagar, J. M. Pérez-Jordá, V. H. Smith Jr. and R. O. Esquivel, Chem. Phys. Lett. 1994, 219, 15.

[6] N. L. Guevara, R. P. Sagar and R. O. Esquivel, Phys. Rev. A 2003, 67, 012507; N. L. Guevara, R. P. Sagar and R. O. Esquivel, J. Chem. Phys. 2003, 119, 7030.

[7] Ch. C. Moustakidis and S. E. Massen, Phys. Rev. B 2005, 71, 045102. 


\section{$Z=18$}
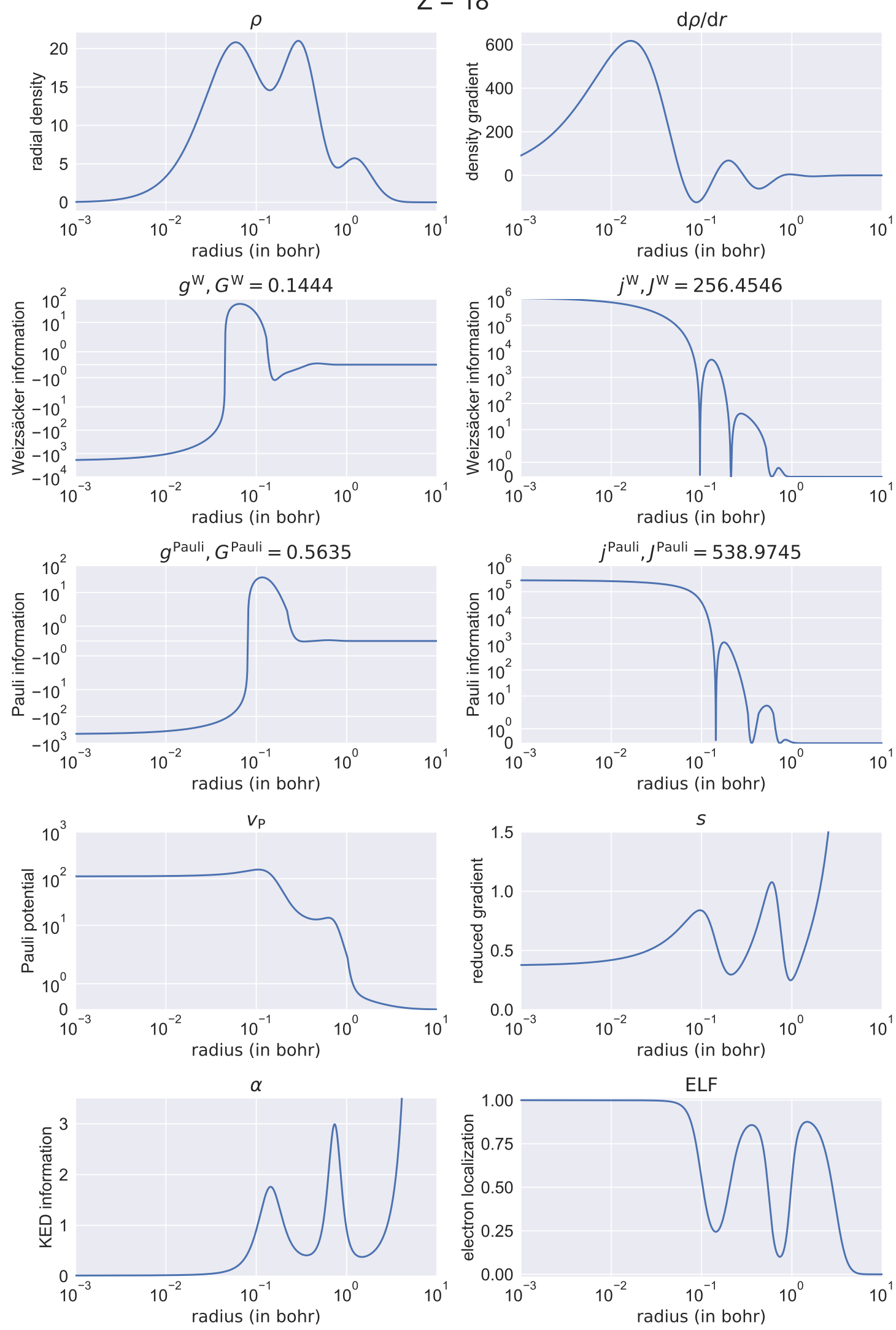

FIG. 3: (Color online) $\varrho, d \varrho / d r, g^{W}, j^{W}, g^{\text {Pauli }}, j^{\text {Pauli }}, v_{P}, s, \alpha$ and $E L F$ as functions of radial distance for the Ar atom in atomic units. 

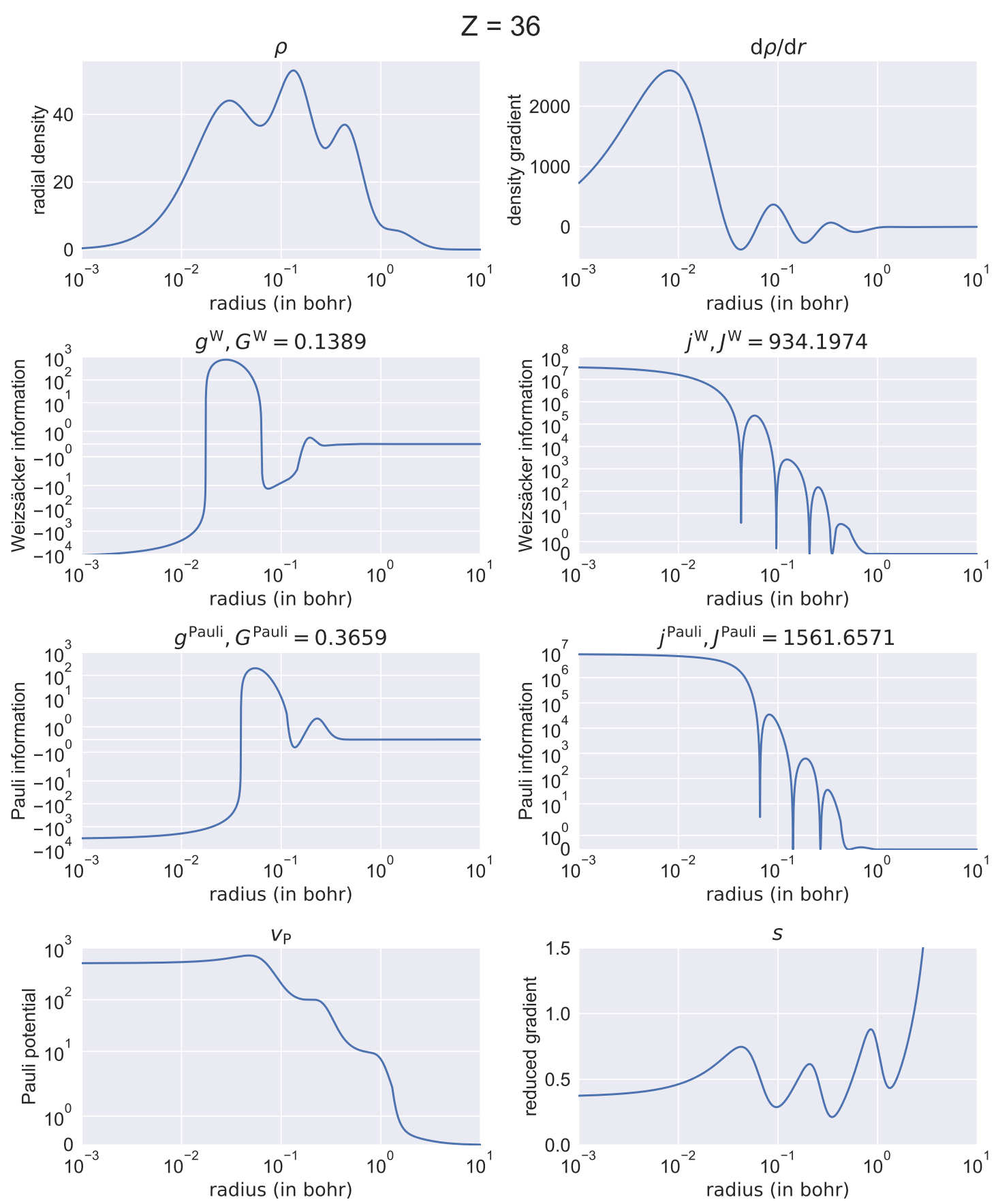

$\alpha$
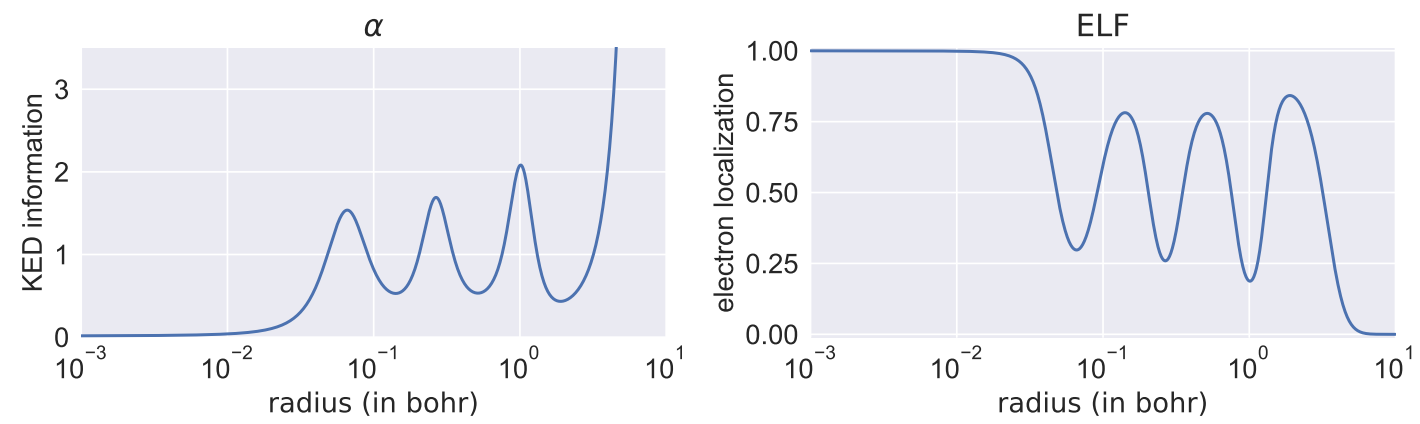

FIG. 4: (Color online) $\varrho, d \varrho / d r, g^{W}, j^{W}, g^{\text {Pauli }}, j^{\text {Pauli }}, v_{P}, s, \alpha$ and $E L F$ as functions of radial distance for the $\mathrm{Kr}$ atom in atomic units. 

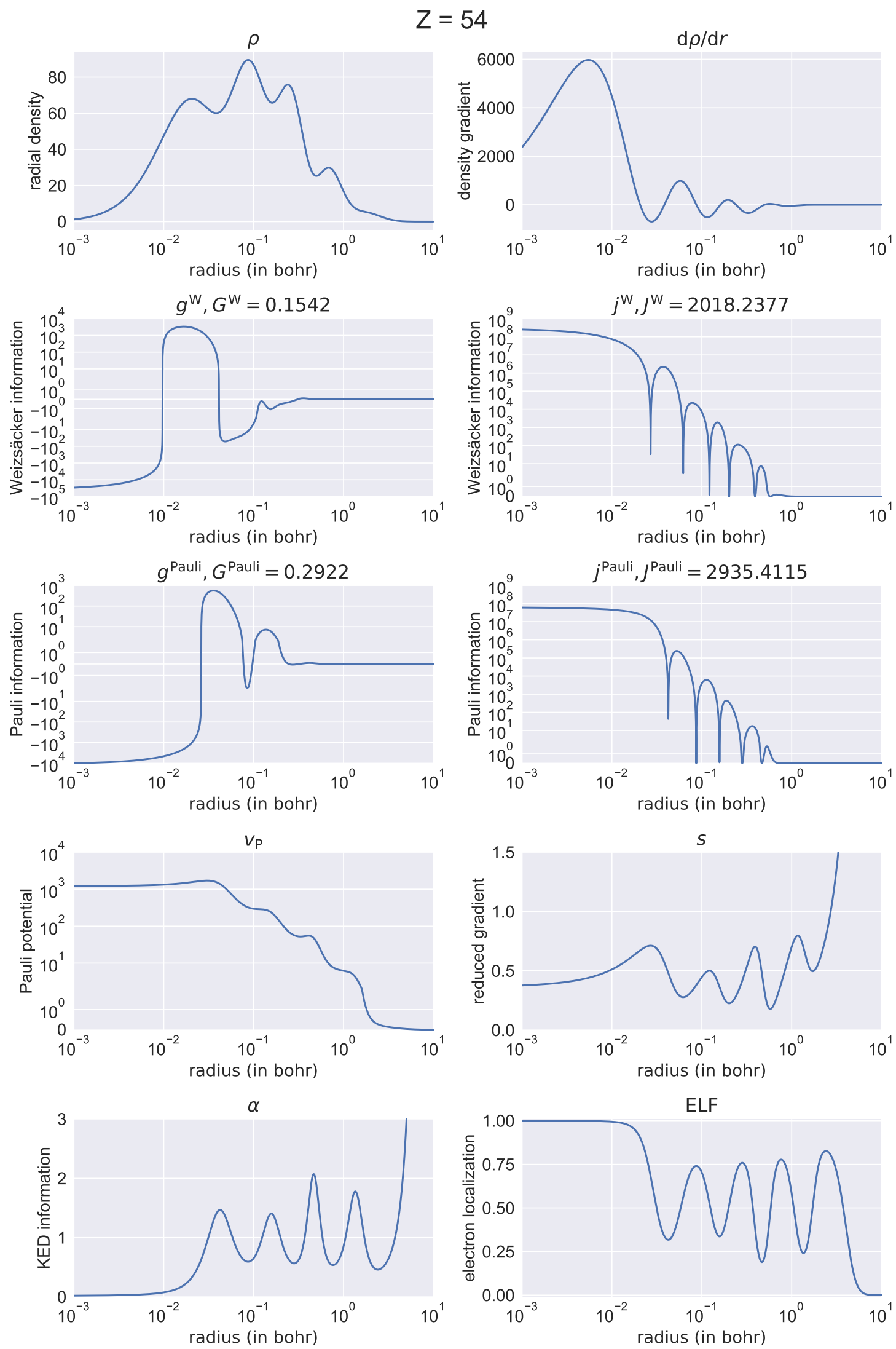

FIG. 5: (Color online) $\varrho, d \varrho / d r, g^{W}, j^{W}, g^{\text {Pauli }}, j^{\text {Pauli }}, v_{P}, s, \alpha$ and $E L F$ as functions of radial distance for the Xe atom in atomic units. 
[8] M. Reginatto, Phys. Rev. A 1998, 58, 1775.

[9] E. Romera, P. Sánchez-Moreno and J. S. Dehesa, Chem. Phys. Lett. 2005, 414, 468.

[10] Á. Nagy, Chem. Phys. Lett. 2006, 425, 154.

[11] Á. Nagy and K. D. Sen, Phys. Lett. A 2006, 360, 291.

[12] I. Hornyák and Á. Nagy, Chem. Phys. Lett. 2007, 437, 132.

[13] E. Romera and J. S. Dehesa, J. Chem. Phys. 2004, 120, 8906.

[14] E. Romera, Mol. Phys. 2002, 100, 3325.

[15] S. B. Liu, J. Chem. Phys. 2007, 126, 191107.

[16] Á. Nagy, Chem. Phys. Lett. 2007, 449, 212.

[17] Á. Nagy and S. B. Liu, Phys. Lett. A 2008, 372, 1654.

[18] J. B. Szabó, K. D. Sen and Á. Nagy, Phys. Lett. A 2008, 372, 2428.

[19] R. F. Nalewajski, Found. Chem. 2014, 16, 27.

[20] C. E. Shannon, Bell Syst. Tech. J. 1948, 27, 379.

[21] S. Kullback and R. A. Leibler, Ann. Math. Stat. 1951, 22, 79; S. Kullback, Statistics and Information Theory, Wiley, New York, 1959; G. Jumarie, Relative Information. Theories and Applications, Springer-Verlag, Berlin, 1990.

[22] R. A. Fisher, Proc. Cambridge Philos. Soc. 1925, 22, 700.

[23] B. R. Frieden, Physics form Fisher Information. A Unification (Cambridge University Press, Cambridge, 1998).

[24] B. R. Frieden, Am. J. Phys. 1989, 5\%, 1004.

[25] R. C. Venkatesan and A. Plastino, Phys. Lett. A 2014, 378, 1341.

[26] Á. Nagy and N. H. March, Mol. Phys. 1997, 90, 271.

[27] M. Kohout, A. Savin and H. Preuss, J. Chem. Phys. 1991, 95, 1928.

[28] G. Toscani, Ric. Mat. 2016, 66, 15.

[29] J. Sun, B. Xiao, Y. Fang, P. Hao, A. Ruzsinszky, G. I. Csonka, G. E. Scuseria and J. P. Perdew, Phys. Rev. Lett. 2013, 111, 106401.

[30] J. Tao, J. P. Perdew, V. N. Staroverov and G. E. Scuseria, Phys. Rev. Lett. 2003, 91, 146401.

[31] J. P. Perdew, J. Tao, V. N. Staroverov and G. E. Scuseria, J. Chem. Phys. 2004, 120, 6898.

[32] J. P. Perdew, S. Kurth, A. Zupan and P. Blaha, Phys. Rev. Lett. 1999, 82, 2544.

[33] J. Sun, A. Ruzsinszky and J. P. Perdew, Phys. Rev. Lett. 2015, 115, 036402.

[34] C. Beck and F. Schlögl, Thernodynamics of chaotic systems: An introduction, Cambridge 
Univ. Press, Cambridge, 1993.

[35] Á. Nagy and E. Romera, Phys. Lett. A 2009, 373, 844.

[36] I. Csiszár, Magyar Tud. Akad. Mat. Kut. Int. Közl. 1963, 8, 85; Stud. Sci. Math. Hung. 1967, 2, 299.

[37] F. Schlögl, Phys. Rep. 1980, 62, 268.

[38] A. D. Becke and K. E. Edgecombe, J. Chem. Phys. 1990, 92, 5397.

[39] A. Savin, B. Silvi and F. Colonna, Can. J. Chem. 1996, 74, 1088.

[40] A. Savin, R. Nesper, S. Wengert and T. F. Fässler, Angew. Chem. Int. Ed. Engl. 1997, 36, 1809.

[41] A. E. Mattsson and J. M. Wills, Int. J. Quantum Chem. 2016, 116, 834.

[42] O. Čertík, J. E. Pask and J. Vackár̆, Comp. Phys. Comm. 2013, 184, 1777.

[43] S.H. Vosko, L. Wilk, and M. Nusair, Can. J. Phys. 1980, 58, 1200. 